\title{
MORFOMETRIA DO RAMO MANDIBULAR EM DIFERENTES TIPOS CRANIOMÉTRICOS
}

\author{
MANDIBULAR BRANCH MORPHOMETRY \\ IN DIFFERENT CRANIOMETRIC TYPES
}

\begin{abstract}
Atson Carlos de Souza Fernandes*, Raquel Pimentel Loureiro**
Autor para correspondência: Atson Carlos de Souza Fernandes - atsonfernandes@yahoo.com.br

*Doutor em Ciências Morfológicas. Pesquisador Colaborador do Departamento de Diagnóstico da UNICAMP.

Professor do curso de Odontologia da Escola Bahiana de Medicina e Saúde Pública.

**Graduada em Odontologia pela Escola Bahiana de Madicina e Saúde Pública
\end{abstract}

INTRODUÇÃO: A mandíbula é um dos ossos da face mais susceptíveis à fraturas e deformidades, tratados através de procedimentos cirúrgicos. Na técnica de osteotomia sagital do ramo mandibular (OSRM) o corte horizontal é feito na face medial do ramo imediatamente acima da língula. OBJETIVO: O propósito desse estudo foi verificar a existência de correlação da altura e largura do ramo em relação ao gênero e índice cefálico. MATERIAL E MÉTODO: Foram utilizadas 48 mandíbulas humanas dentadas de adultos do tipo dolico (16), meso (16) e braquicefálico (16). Os pontos pré determinados utilizados como referências anatômicas no ramo para mensurações foram: (I) ponto mais inferior da incisura mandibular, (B) ponto mais alto da margem inferior, (A) ponto mais posterior da margem anterior (A) e (P) ponto mais anterior da margem posterior. RESULTADOS: A média da altura e largura do ramo mandibular não apresentou diferença na comparação entre os índices cefálicos (dolico 47,05 / 32,09mm; meso - 47,27 / 32,57mm; braquicefálico - 46,41 / 29,58mm). Na comparação das dimensões com o gênero, a diferença encontrada foi a altura do ramo no sexo masculino $(49,08 \mathrm{~mm})$, maior do que no feminino $(42,76 \mathrm{~mm})$. CONCLUSÕES: Os resultados aqui encontrados podem ser utilizados como referência em procedimentos cirúrgicos do ramo mandibular, investigações forenses e estudos de comparação a dados obtidos em espécimes de outras nacionalidades.

PALAVRAS-CHAVE: Cirurgia; Mandíbula; Anatomia. 
INTRODUCTION: The jaw is one of the facial bone most susceptible to fracture and deformities that must be treated with surgical procedures. On the sagittal split osteotomy of mandibular ramus technique (SSOMR) the horizontal cut is done on ramus medial aspect immediately above the lingula. OBJECTIVE: The aim of this study was to verify the possible correlation of the height and width of the ramus with the gender and cephalic index. MATERIALS AND METHODS: 48 adult, dentate, human mandibles were used, being dolicho- (16), meso- (16) and brachycephalic (16) specimens. The anatomic references on the ramus were: the most inferior point of the mandibular notch (I), the highest point of the inferior board (B), the most posterior point of the anterior margin, $(P)$ the most anterior point of the posterior margin. RESULTS: The mean of the height and width of the mandibular ramus showed no difference compared with the cephalic index (dolicho-47,05/32,09mm; meso-47,27/32,57mm; brachicephalic $-46,41$ / 29,58mm). Assessing the dimensions in light of the individuals gender showed that there was a height difference of the ramus in male individuals $(49,08 \mathrm{~mm})$ that was higher than in female individuals $(42,76 \mathrm{~mm})$. CONCLUSIONS: We believe our results should be used as reference in mandibular ramus surgical procedures, forensic investigations and morphometric comparisons with results of further studies in individuals from other nations.

KEY WORDS: Surgery; Jaw; Anatomy. 


\section{INTRODUÇÃO}

No ramo da mandíbula estão localizadas importantes estruturas anatômicas a exemplo do canal da mandíbula que tem início a partir do forame mandibular, uma ampla abertura próxima à língula, onde está inserido o ligamento esfenomandibular ${ }^{1}$. A língula tem sido utilizada como ponto de referência para a realização da cirurgia ortognática, bem como para técnica de procedimento anestésico, mudando de posição com a idade. $\mathrm{Na}$ técnica de osteotomia sagital, a posição da língula serve como referência para a realização do corte horizontal que deve ser realizado imediatamente acima dessa estrutura anatômica ${ }^{2,3}$.

A osteotomia sagital dos ramos mandibulares (OSRM) é o procedimento cirúrgico mais utilizado em cirurgia ortognática. Introduzida por Schuchardt, em 1942, em 1957 foi popularizada por Trauner e Obwegeser, e modificada por DalPont, tornando-se o procedimento de eleição em cirurgias ortognáticas na mandíbula ${ }^{4,5}$. A técnica permite o avanço e recuo mandibular, proporcionando uma melhora da função mastigatória, da fonética e da harmonia facial do paciente ${ }^{2}$. Sua técnica cirúrgica consiste em um corte horizontal na face medial do ramo da mandíbula que é feito logo acima da língula ${ }^{6}$. Entretanto, é importante notar que a forma de apresentação da língula pode variar entre indivíduos e até mesmo entre os lados de uma mesma pessoa, o que implica em risco constante de lesão do nervo alveolar inferior ${ }^{7,8,9}$. Somado a isso, observa-se $\circ$ fato de que são poucos os trabalhos que detalham as estruturas anatômicas e o risco de lesão das mesmas quando relacionadas à procedimentos cirúrgicos da OSMR $^{10}$.

A cefalometria é uma técnica que estuda as complexidades anatômicas da cabeça de um ser humano, sendo o índice cefálico uma das dimensões cefalométricas mais utilizadas. As formas da cabeça são classificadas em quatro categorias internacionais, sendo estas: dolicocéfalo, braquicéfalo, mesocéfalo e hiperbraquicéfalo" ${ }^{1}$.

Com base em estudos anteriores, observa-se na grande maioria dos trabalhos que as estruturas anatômicas relacionadas à osteotomia sagital do ramo mandibular são pouco relatadas ${ }^{11}$. O objetivo desse estudo é identificar a influência dos tipos craniométricos e do gênero no comprimento e largura do ramo de mandíbulas humanas, determinando a possível existência de um padrão anatômico que possa influenciar em procedimentos de interesse clínico-cirúrgicos.

\section{METODOLOGIA}

Este estudo recebeu a aprovação do Comitê de Ética em Pesquisa da Escola Bahiana de Medicina e Saúde Pública, Bahia, Brasil (CAAE N. 30717214.2.0000.5544). Foram utilizadas 48 hemi-mandíbulas humanas, de indivíduos adultos, secas e dentadas, de origem sul-americana,

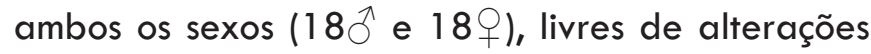
macroscópicas, obtidas de espécimes com diferentes índices cefálicos: dolicocefálico (16), braquicefálico (16) e mesocefálico (16), pertencentes ao acervo de ossos do Laboratório de Anatomia da Escola Bahiana de Medicina e Saúde Pública e da Universidade Federal de São Paulo.

O método utilizado para identificação dos pontos de referência para cálculo das distâncias foi o mesmo descrito no trabalho de Fernandes et al (2015) ${ }^{12}$.

Os marcos ósseos selecionados foram: o ponto mais baixo da incisura mandibular (I); o ponto mais alto da margem inferior do ramo mandibular (B); o ponto mais posterior à margem anterior do ramo mandibular $(A)$; o ponto mais anterior na margem posterior do ramo mandibular (P) (Fig. 01).

Com a hemi-mandíbula repousada em uma superfície plana, horizontal, com a face medial voltada para cima, uma câmera digital (CANON EOS Rebel T3i, fabricada em Taipé, Taiwan) foi posicionada 15 centímetros acima do mesmo, visando o aspecto medial do ramo. A foto foi tirada com a margem posterior do ramo posicionado em uma linha vertical simulando a posição da abertura de boca.

Com uma linha reta horizontal digital traçada ao longo da imagem capturada foi possível identificar em cada amostra os pontos (I) e (B). Da mesma forma, com uma linha vertical digital e traçada ao longo da imagem capturada foi possível identificar os pontos (A) e (P) (Fig. 1).

As imagens tomadas dos ramos das mandíbulas estudadas, tem como objetivo identifica e padronizar 
a posição exata dos reparos ósseos na imagem e na peça estudada para realização da mensuração do ramo da mandíbula. Dessa forma diminuir as chances de erro ao tomar as medidas do ramo.

Figura 1. Identificação dos pontos anatômicos de referência para estudo $(A, P, B$ e $I)$, no ramo da mandíbula

As mensurações das hemi-mandíbulas foram realizadas de acordo com os pontos prédeterminados nas peças, a partir das referências obtidas através das imagens. Todas as medições foram realizadas pela mesma pessoa usando um paquímetro digital (Mitutoyo - Mycal Absoluto série 500 - 500-144 B) e as distâncias foram sempre medidas em milímetros.

As seguintes distâncias foram mensuradas a partir dos pontos IB verticalmente, e no sentido horizontal, AP. Assim obtendo os valores da altura e largura do ramo, respectivamente.

\section{ANÁLISE ESTATÍSTICA}

Para comparar a altura e largura do ramo da mandíbula entre os índices cefálicos, a análise paramétrica unidirecional de variância (ANOVA) foi utilizada e calculado o tempo de chegada ao quadrado.

A correlação da altura e largura da mandíbula de acordo com o sexo foi calculada através do método Cohen's d statistic.

As análises foram realizadas através do $R$ statistical package version $\mathrm{R}$ 3.2.2 (2015).

\section{RESULTADOS}

\section{TIPO CRANIOMÉTRICO}

A comparação da altura (I/B) do ramo da mandíbula com os diferentes índices cefálicos pode ser observada na Tabela 1. Não houve diferença estatisticamente significante da altura do ramo da mandíbula entre os índices cefálicos (dolicocefálico, mesocefálico, braquicefálico), $(p=0,92)$.

Tabela 1. Comparação da altura com o tipo craniométrico

\begin{tabular}{llcccccc}
\hline Mensuração & N & Tipo Craniométrico & Média & SD. & Min. & Máx. & p* \\
\hline \multirow{3}{*}{ Altura } & 16 & Dolicocéfalo & 47,05 & 5,97 & 36,88 & 57,68 & \\
& 16 & Mesocéfalo & 47,27 & 4,41 & 37,94 & 54,02 & 0,92 \\
& 16 & Braquicéfalo & 46,41 & 7,93 & 22,47 & 58,01 & \\
\hline
\end{tabular}

Na comparação da largura entre os índices cefálicos, a dimensão do tipo craniométrico braquicefálico se mostrou menor quando comparada aos demais, apesar de não haver expressividade estatística $(p>0,05)$. As médias encontradas nos tipos craniométricos dolicocefálico, mesocefálico e braquicefálico foram, respectivamente, $32,09,32,57 \mathrm{~mm}$ e $29,58 \mathrm{~mm}$ (Tabela 2).

Tabela 2. Comparação da largura com o tipo craniométrico

\begin{tabular}{lccccccc}
\hline Mensuraçã。 & N & Tipo Craniométrico & Média & SD. & Min. & Máx. & p* \\
\hline \multirow{3}{*}{ Altura } & 16 & Dolicocéfalo & 32,09 & 4,09 & 26,50 & 42,34 & \\
& 16 & Mesocéfalo & 32,57 & 3,02 & 28,80 & 40,40 & 0,064 \\
& 16 & Braquicéfalo & 29,58 & 4,46 & 23,60 & 39,90 & \\
\hline
\end{tabular}


No sexo masculino o resultado da comparação da altura do ramo $(49,08 \mathrm{~mm})$ foi significativamente maior em relação a altura encontrada no sexo feminino $(42,76 \mathrm{~mm})$, onde o valor de $p$ foi de 0,0013 (Tabela 3).

Tabela 3. Comparação da altura com o gênero

\begin{tabular}{lccccccc}
\hline Mensuração & N & Tipo Craniométrico & Média & SD. & Min. & Máx. & p* \\
\hline \multirow{2}{*}{ Altura } & 18 & $M$ & & & & & \\
& 18 & F & 49,08 & 4,79 & 37,94 & 58,01 & $0,0013^{* *}$ \\
& 18 & 42,76 & 6,63 & 22,47 & 51,75 & \\
\hline *Cohen's d statistic; **estatisticamente significante & & & & & &
\end{tabular}

Na análise da largura do ramo não houve prevalência estatisticamente significativa do sexo masculino $(31,96 \mathrm{~mm})$ quando comparado ao sexo feminino $(30,26 \mathrm{~mm}),(p=0,12)$, (Tabela 4).

Tabela 4. Comparação da largura com o gênero

\begin{tabular}{|c|c|c|c|c|c|c|c|}
\hline Mensuração & $\mathbf{N}$ & Tipo Craniométrico & Média & SD. & Min. & Máx. & $\mathbf{p}^{*}$ \\
\hline & 18 & $M$ & 31,96 & 4,3 & 23,60 & 42,34 & \\
\hline \multirow[t]{2}{*}{ Altura } & & & & 9 & & & 0,12 \\
\hline & 18 & $\mathbf{F}$ & 30,26 & $\begin{array}{r}3,2 \\
4\end{array}$ & 25,32 & 34,70 & \\
\hline
\end{tabular}

\section{DISCUSSÃO}

Para a realização da osteotomia sagital do ramo mandibular preconiza-se um planejamento cuidadoso e uma execução meticulosa dessa técnica. É um procedimento delicado que tem como ponto de referência a língula na realização do corte horizontal ${ }^{2}$. A língula por sua vez é uma estrutura que apresenta variações na sua forma e tamanho, ocasionando riscos de lesão a importantes estruturas nervosas durante a realização da técnica ${ }^{7,8}$.

A literatura mostra diversos trabalhos relacionados ao estudo das dimensões do ramo da mandíbula ${ }^{5,12-16}$. Com relação a altura do ramo, os valores encontrados por Fernandes et al (12) foram $49,49 \mathrm{~mm}$ (dolicocéfalo), 48,25mm (mesocéfalo) e $48,01 \mathrm{~mm}$ (braquicéfalo). Ennes e Medeiros 13 encontrou $46,6 \mathrm{~mm}$ do lado direito e $48,8 \mathrm{~mm}$ do lado esquerdo. Valente et $\mathrm{al}^{15}$ também considerou os lados, encontrando $64 \mathrm{~mm}$ do lado direito e $63,65 \mathrm{~mm}$ do lado esquerdo. Fontoura et $\mathrm{al}^{14} \mathrm{e}$
Monazzi et $a l^{5}$, independente de lado, gênero ou índice cefálico calcularam a altura do ramo em $49,4 \mathrm{~mm}$ e $42,84 \mathrm{~mm}$, respectivamente. No presente estudo, as médias das alturas do ramo mandibular (IB) quando analisadas junto ao índice cefálico foram $47,05 \mathrm{~mm}$ (dolicocéfalo), 47,27 mm (mesocéfalo) e $46,41 \mathrm{~mm}$ (braquicéfalo), valores semelhantes àqueles encontrados nos estudos anteriores, com exceção dos valores apresentados no estudo de Valente et al. Essa diferença pode ser justificada através da metodologia utilizada pelo autor, que utilizou mandíbulas parcialmente dentadas e tomou, como ponto de referência para realização das mensurações do ramo, reparos ósseos diferentes dos pontos identificados nesse estudo. Não houve diferença estatística entre os índices cefálicos, da mesma forma que não foi encontrada no trabalho de Fernandes et al, $2015^{12}$.

No estudo sobre a largura do ramo, as médias encontradas no presente estudo (AP) foram 32,09 mm, $32,57 \mathrm{~mm}$ e $29,58 \mathrm{~mm}$ para dolicocéfalo, mesocéfalo e braquicéfalo, respectivamente, sendo a dimensão 
encontrada no tipo craniométrico braquicefálico (tabela II) menor quando comparada aos demais. Tais achados se assemelham àqueles encontrados em Fernandes et al ${ }^{12}$ cujas medidas foram $32,40 \mathrm{~mm}$ (dolicocefálico), $31,82 \mathrm{~mm}$ (mesocefálico) e $30,44 \mathrm{~mm}$ (braquicefálico). Os números também se aproximam dos valores encontrados em Monazzi et al5, 32,02mm, e Fontoura et $\mathrm{al}^{14}, 31,03 \mathrm{~mm}$. No estudo de Ennes e Medeiros 13 os valores encontrados da largura do ramo nos lados direito $(26,70 \mathrm{~mm})$ e esquerdo $(26,9 \mathrm{~mm})$ divergiram daqueles encontrados na presente investigação, assim como aos demais. É possível que esta diferença esteja relacionada à metodologia utilizada pelos autores que consideraram mandíbulas desdentadas totais e parciais, ao contrário dos nossos modelos (mandíbulas dentadas totais), assim como no trabalho de Fernandes et $\mathrm{al}^{12}$, Fontoura et $\mathrm{al}^{14} \mathrm{e}$ Monazzi et $a^{5}$. Além disso, no estudo de Ennes e Medeiros ${ }^{13}$ não foi considerada a distância entre as margens anterior e posterior do forame da mandíbula no cálculo da largura do ramo.

Quando comparada a altura entre os gêneros foi observado no presente estudo que o sexo masculino $(49,08 \mathrm{~mm})$ apresentou uma média significativamente maior quando comparado ao sexo feminino $(42,76 \mathrm{~mm})$. Valores semelhantes foram encontrados no estudo de Medeiros et $\mathrm{al}^{16}$, com mesma diferença entre os sexos: gênero masculino $49,6 \mathrm{~mm}$ (lado direito) e $48,88 \mathrm{~mm}$ (lado esquerdo), e gênero feminino $44,37 \mathrm{~mm}$ (lado direito) e $43,14 \mathrm{~mm}$ (lado esquerdo). No estudo de Olate et al17 os valores encontrados para altura do ramo (11,6mm - masculino e 10,8mm - feminino) foram inferiores aos apresentados nesta pesquisa, assim como aos achados de Medeiros et $a^{16}$. É possível que essa diferença esteja relacionada ao fato dos autores terem realizado a mensuração em imagens de radiografia panorâmica.

$\mathrm{Na}$ comparação da largura entre os gêneros $(31,96 \mathrm{~mm}$ - masculino e $30,26 \mathrm{~mm}$ - feminino) este estudo não encontrou diferença significativa. Já no trabalho de Medeiros et $\mathrm{al}^{16}$, apesar dos valores encontrados no lado direito terem sido semelhantes aos nossos no gênero masculino $(29,33 \mathrm{~mm}$ lado direito e $29,34 \mathrm{~mm}$ lado esquerdo), no gênero feminino os valores encontrados foram significativamente menores $(26,32 \mathrm{~mm}$ - lado direito e $26,43 \mathrm{~mm}$ - lado esquerdo).

\section{CONSIDERAÇÕES FINAIS}

Acredita-se que os valores obtidos nesse estudo e as diferenças encontradas na comparação entre os gêneros e tipos craniométricos possam servir de referência para a realização de procedimentos cirúrgicos da região pesquisada com maior segurança, estudos forenses e comparações morfométricas de investigações futuras entre populações.

\section{AGRADECIMENTO}

À FAPESB (Fundação de Amparo à Pesquisa do Estado da Bahia) pelo apoio à realização deste estudo

\section{REFERÊNCIAS}

1. Madeira MC. Vascularização sanguínea e linfática da face. In: Madeira MC. Anatomia da face: bases anatomofuncionais para a prática odontológica. São Paulo:Sarvier;1998. P. 153-77.

2. Polido WD. Osteotomia sagital do ramo mandibular. In: Araújo A. Cirurgia ortognática. São Paulo:Santos; 1999. P. 113-30.

3. Kim HJ, Lee HY, Chung IH, Cha IH, Yi CK. Mandibular anatomy related to sagittal split ramus osteotomy in Koreans. Yonsei Med J. 1997;38(1):19-25. doi: 10.3349/ ymj.1997.38.1.19

4. Cillo JE, Stella JP. Selection of sagittal split ramus osteotomy technique based on skeletal anatomy and planned distal segment movement: current therapy. J Oral Maxillofac Surg. 2005;63(1):109114.

5. Monnazzi MS, Passeri LA, Gabrielli MFR, Bolini PDA, De Carvalho WRS, Da Costa Machado 
H. Anatomic study of the mandibular foramen, lingula and antilingula in dry mandibles, and its statistical relationship between the true lingula and the antilingula. Int J Oral Maxillofac Surg. 2012;41:74-8. doi: 10.1016/i.ijom.2011.08.009

6. Tom WK, Martone CH, Mintz SM. A study of mandibular ramus anatomy and its significance to sagittal split osteotomy. Int J Oral Maxillofac Surg. 1997;26(3):176-8.

7. Jansisyanont $P$, Apinhasmit W, Chompoopong $S$. Shape, height and location of the lingual for sagittal ramus osteotomy in Thais. Clin Anat. 2009;22(7):787-93. doi: 10.1002/ca.20849

8. Kositbowornchai S, Siritapetawee $M$, Damrongrungruang T, Khongkankong W, Chatrchaiwiwatana S, Khamanarong $K$ et al. Shape of the lingual and its localization by panoramic radiograph versus dry mandibular measurement. Surg Radiol Anat. 2007;29(8):689-94. doi: $10.1007 / \mathrm{s} 00276-007-0270-9$

9. Fernandes ACS, Fraga TL, Neto AIT, Freitas AC. Delimitação morfométrica para o corte horizontal da osteotomia sagital no ramo mandibular. Rev Cir Traumatol buco-maxilo-facial. 2009;9(2):107-14.

10. Balcioglu HM, Kilic C, Varol A, Ozan H, Kocabiyik N, Yildirim M. A Morphometric Study of the Maxillary Artery and Lingula in Relation to Mandibular Ramus Osteotomies and TMJ Surgery. Eur J Dent. 2012;4(2):166-70.

11. Golalipour MJ, Jahanshashi M, Haidari K. Morphological evalution of head in Turkman males in Gorgan-Northof Iram. Int J Morphol. 2007;25(1):99-102.

12. Fernandes ACS, Loureiro RP, Oliveira L, De Moraes M. Mandibular foramen location and lingula height in dentate dry mandibles, and its relationship with cephalic index. Int. J. Morphol. 2015;33(3):1038-1044. doi: 10.4067/S071795022015000300037

13. Ennes JP, Medeiros RM. Localization of mandibular foramen and clinical implications. Int. J. Morphol. 2009;27(4):1305-1311. doi: 10.4067/ S0717-95022009000400053
14. Fontoura RA, Vasconcellos HA, Campos AES. Morphologic basis for the intraoral vertical ramus osteotomy: anatomic and radiographic localization of the mandibular foramen. J Oral Maxillofac Surg. 2002;60(2):660-665.

15. Valente VB, Arita WM, Gonçalves PCG, Campos JADB, Capote TSO. Location of the mandibular foramen according to the amount of dental alveoli. Int. J. Morphol. 2012;30(1):77-81.

16. Júnior MDM. Localização anatomo-topográfica do forame mandibular em mandíbulas maceradas de adultos [dissertação]. Recife: Universidade Federal de Pernambuco; 2002.

17. Olate S, Pozzer L, Sawazaki R, Asprino L, Moraes $M$. ¿̇Existen diferencias en la morfometria mandibular de pacientes candidatos a cirugía ortognática?. Parte 2: Influencias del género. Int. J. Morphol. 2009;27(3):777-781. doi: 10.4067/ S0717-95022009000300023. 